III.

Aus dem pharmakologisehen Institut der Universität Wien.

\title{
Zur Pharmakologie der Wärmenarkose des Kaltblüterherzens.
}

(Ausgeführt mit Unterstuitzung der Fürst Liechtenstein-Spende.)

$$
\text { Von }
$$

Dr. Cäsar Amsler und Prof. Dr. Ernst P. Pick, Assistenten am Institut.

(Mit 10 Kurven.)

Die Erscheinung, daß der Ventrikel des Froschherzens beim Erwärmen auf eine bestimmte Temperatur in völligen diastolischen Stillstand verfällt, um beim Abkühlen seine normale Tätigkeit wieder zu erlangen, ist dem Phänomen der Narkose so sehr ähnlich, daß man sie wohl ohne weiteres als Wärmenarkose des Herzens anffassen darf. Bereits CI. Bernard (1) hat die durch Wärme am Froschmuskel erzengte und durch Abküillung reversible Nervenlähmung als durch Anästhesie bedingt gedeutet, und Hans Meyer (2) hat den beim Erwärmen des ganzen Frosches eintretenden schlafähnlichen Zustand, welcher durch Abkühlen des Tieres reversibel ist, als Wärmenarkose bezeichnet. Wiewohl seit den Untersuchungen von Schelske (3) nnd Cyon(4) in den $60 \mathrm{er}$ Jahren des vorigen Jahrhunderts eine groBe Literatur über den Einfluß erhöhter Temperatur auf das Froschherz entstanden ist, liegen über die Ursache des in Frage stehenden Wärmestillstandes nicht viele Untersuchungen vor. So faßte Cyon, ausgebend von der Vorstellung, daß plötzliches Erwärmen des Herzens von 20 auf $40^{\circ} \mathrm{C}$ die nervösen Herzteile reize, den Wärmestillstand als Erregung der vagalen Hemmungsapparate auf. Da jedoch Aristow (5) dieselbe Erscheinung auch am atropinisierten Herzen beobachtete, nahm letzterer an, daß die erhöhte Temperatur die Kontraktionsfähigkeit des Muskels und die Tätigkeit der Nervenzellen des Herzens herabsetzt. Dieselbe Ansicht äußerten Ludwig und Luch- 
singer (6), während Stewart (7) aus der Beobachtung, daß die ganglienfreie Herzspitze auf Erwärmen genan so reagiert, wie der ganze Ventrikel, den Schluß zog, daß der Wärmestillstand des Herzens bedingt ist nicht dürch Lähmung der motorischen Ganglien, sondern durch Versagen der Muskulatur. Ide (8) nahm als Ursache eine reversible Änderung in der stofflichen Zusammensetzung des Herzmuskels an. Endlich hat Unger (9) beobachtet, daß an dem durch Stanniussche Ligatur zwischen dem Sinus und den Vorhöfen stillgestellten, auf eine bestimmte Temperatur erwärmten und im Bereich des Ventrikels elektrisch gereizten Herzen sich nur der Ventrikel auf den Reiz hin zusammenzog, die Vorhöfe aber in Ruhe verharrten (Unterbrechung der umgekehrten Reizleitung nach Engelmann). Daraus zog er den SchluB, daß der Wärmestillstand auf Schädigung des Reizleitungssystems beruht. Aus diesen Literaturangaben geht hervor, daß die Ursache des Wärmestillstandes von den verschiedenen Autoren ganz verschieden gedeutet, und die Schädigung fast aller Herzqualitäten für das Eintreten desselben verańtwortlich gemacht worden ist. Unsere friiheren Beobachtungen am lichtempfindlich gemachten Herzen (10) erbrachten den Beweis, daß das Reizleitungssystem für den Lichtschaden am empfindlichsten ist, und es schien uns daher nicht unmöglich, daß der durch Wärmenarkose erzeugte Ventrikelstillstand in erster Linie durch Aufhebung der atrioventrikulären Reizleitung bedingt ist, wie denn von den verschiedenen Herzqualitäten die Reizleitung durch Gifte überhaupt am leichtesten und daher zuerst geschädigt werden zu können scheint. Der Irreversibilität der Lichtschädigung und der völligen Reversibilität der Wärmenarkose kommt hierbei keine prinzipielle Bedeutung zu, da dieser Unterschied nur eine Folge verschiedener Dosierung ist.

\section{Methodik.}

Die Methodik unserer Versuche lehnt sich eng an die für unsere frühere Arbeit beniitzte Versuchsanordnung an und gestaltete sich folgendermaßen: Das nach Straub suspendierte und mit Sauerstoff versehene Froschherz (wir verwendeten fast nur Eskulentenherzen) erwärmten wir in der Weise, daß wir den das Herz umgebenden Wassermantel (s. die Abbildung in unserer eben erwähnten Mitteilung:) durch eine Warmwasserschlange aus Glas heizten. Zugleich wurde die das Herz speisende Ringerlösung erwärmt, indem durch eine in die Trichterkanüle eingefïhrte U-förmig gebogene Glasröhre warmes Wasser floß. Beide Leitungen (Gummischläuche) wurden aus einem Ostwaldschen Warmwasserbad, dessen. Inhalt konstant bei $80^{\circ} \mathrm{C}$ 
gehalten war, gespeist. Der Wassermantel wurde fortwährend erwärmt, so daß die Temperatur der das Herz unmittelbar umgebenden Luft eine konstante Höhe von etwa $38^{\circ} \mathrm{C}$ anfwies. Zur Abkühlung der Ringerlösung diente ein Gefäß mit kaltem Wasser, welches mit der durch das U-Robr fuhrenden Leitung verbunden war. Sowohl die Temperatur der das Herz umgebenden Luft, wie der Nälirlösung ließ sich durch Quetschschrauben leicht regulieren und wurde darch dauernd eingeführte Thermometer gemessen. Die Trichterkanüle und das die Luftwärme messende Thermometer. waren in einem flachen Kork befestigt, der seinerseits den Raum innerhalb des Wärmemantels, in welchem das Herz hing, verschloß. Die Herzbewegungen wurden in tublicher Weise anf ein Kymographion übertragen.

\section{Über die den Wärmestillstand auslösende Temperatur.}

In der Regel entwickelt sich der Stillstand des Herzens derart, daß bei einer bestimmten Temperatur der Nährflüssigkeit, in unseren Fällen fast immer um $38^{\circ} \mathrm{C}$ herum, der Ventrikel in Diastole stillsteht, während Vorhöfe und Sinus noch mehr oder weniger regelmäßig weiterpulsieren. Hier und da kommt es jedoch vor, daß dabei das ganze Herz aussetzt. Diese Temperatur ist genau dieselbe, bei welcher die Wärmenarkose des ganzen Frosches eintritt. Die Angaben anderer Autoren, daß sich der Wärmestillstand schon bei niedrigerer, oder erst bei höherer Temperatur einstellt (Flatow 11 z. B. beobachtete das Einsetzen des Wärmeschocks zwischen 32 und $35^{\circ} \mathrm{C}$, Ide and Aristow bei $50^{\circ} \mathrm{C}$ ), sind durch die Versuchsanordnung begrtindet und entsprechen nicht den tatsächlichen Verhältnissen. Auffälligerweise liegen die von Straub(12) am glattmuskeligen Aplysienherzen festgestellten Schocktemperaturen iuber $40^{\circ}$. Stewart beobachtete bei der Schildkröte Ventrikelstillstand bei $49^{\circ} \mathrm{C}$, während die Vorhöfe noch unverändert weiterschlugen.

Entwickelt sich der Wärmestillstand beinahe immer bei der angegebenen Temperatur, so braucht die Tätigkeit des Ventrikels beim Abkühlen nicht sofort unterhalb derselben wieder einzusetzen, sondern kann erst bei Temperaturgraden zurückkehren, bei denen ursprïnglich noch gar keine wesentlichen Veränderungen der Herztätigkeit beobachtet wurden. Nach Schelske setzt die Herzfunktion erst wieder bei einer Temperatur von $14^{\circ} \mathrm{C}$ ein, eine Beobachtung, die auch von anderen gemacht worden ịst. Zuweilen șahen auch wir, daß sich der Ventrikel erst bei niedrigeren Temperaturgraden wieder erholte. Zumeist jedoch setzte die regelmäBige Tätigkeit zwischen 30 und $35^{\circ}, \mathrm{C}$. wieder ein. 


\section{Analyse des Wärmestillstandes.}

1. Mechanische und elektrische Erregbarkeit des stillstehenden Ventrikels.

Das im folgenden angewandte Verfahren zur Analyse des Wärmestillstandes ist im allgemeinen dasselbe, wie wir es zur Analyse des Lichtschocks anwendeten. 'Die einfache Beobachtung lehrt, daß in den allermeisten Fällen, wie es auch Unger sah, während des Stillstandes des Ventrikels Sinus und Vörthöfe ibre regelmäßige Schlagfolge mehrminder beibehalten. Diese Erscheinung wies in Analogie zu unsereren Erfahrungen am lichtgeschädigten Herzen von vornherein auf die vorzügliche Beteiligung des Reizleitungssystems an dem Ventrikelstillstand hin. Wie aus Kurve 1 ersichtlich ist, kann

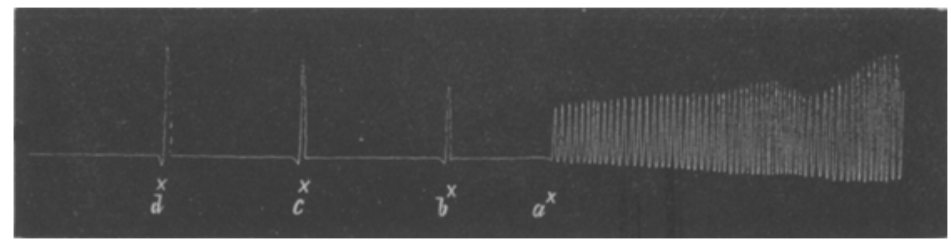

Karve 1. Bei a Eintreten des Ventrikels in den diastolischen Stillstand bei einer Temperatur von $38,5^{\circ} \mathrm{C}$. $b$ Mechanisebe, $c$ und $d$ elektrisehe Reizung des Veatrikels mit Öfnungsinduktionsstrom bei $5 \mathrm{~cm}$ Rollenabstand. Temperatur konstant $38,5^{\circ} .^{\circ} \mathrm{C}$.

der eben in Diastole eingetretene Ventrikel sowohl mechanisch, wie durch die schwächsten, das normale Herz eben noch beeinflussenden Öffnungsinduktionsschläge unverändert erregbar seiṇ. Allerdings pflegt die Erregbarkeit des Muskels auf Induktionsströme und mechanische Reize ohne weitere Steigerung der Temperatur bald und allmählich abzunehmen (s. Kurve 2b). Daraus geht hervor, daß nach und nach die Schädigung von der Reizleitung auch auf die automatischen motorischen Ganglien des Ventrikels und dessen Muskulatur iibergreift, ohne jedoch, was wichtig ist, die Reversibilität durch Abkühlen zu verlieren. Gerade Kurve 2 b zeigt, daß ein bis zum Nichtansprechen auf elektrische Reize durch Wärme geschädigter Ventrikel schon beim Abkühlen auf Temperaturen, die noch keine regelmäßige Herztätigkeit auszulösen imstande sind, die elektrische Reizbarkeit völlig wieder erlangt. Dab die Erregbarkeit des stillstehenden Ventrikels nach nicht zu langer Dauer der Erwärmung noch ganz intakt sein kann, zeigt auch die Tatsache, daß ein einziger Induktionsschlag manchmal, bei stets 


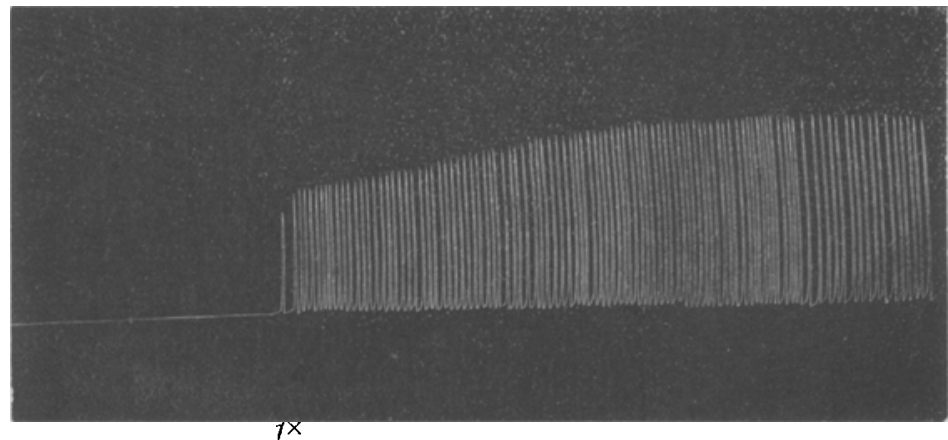

$1^{x}$

Kurve 2a: Bei $1^{\times} 38^{\circ} \mathrm{C}$, Eintreten des Wïrmestillstandes.

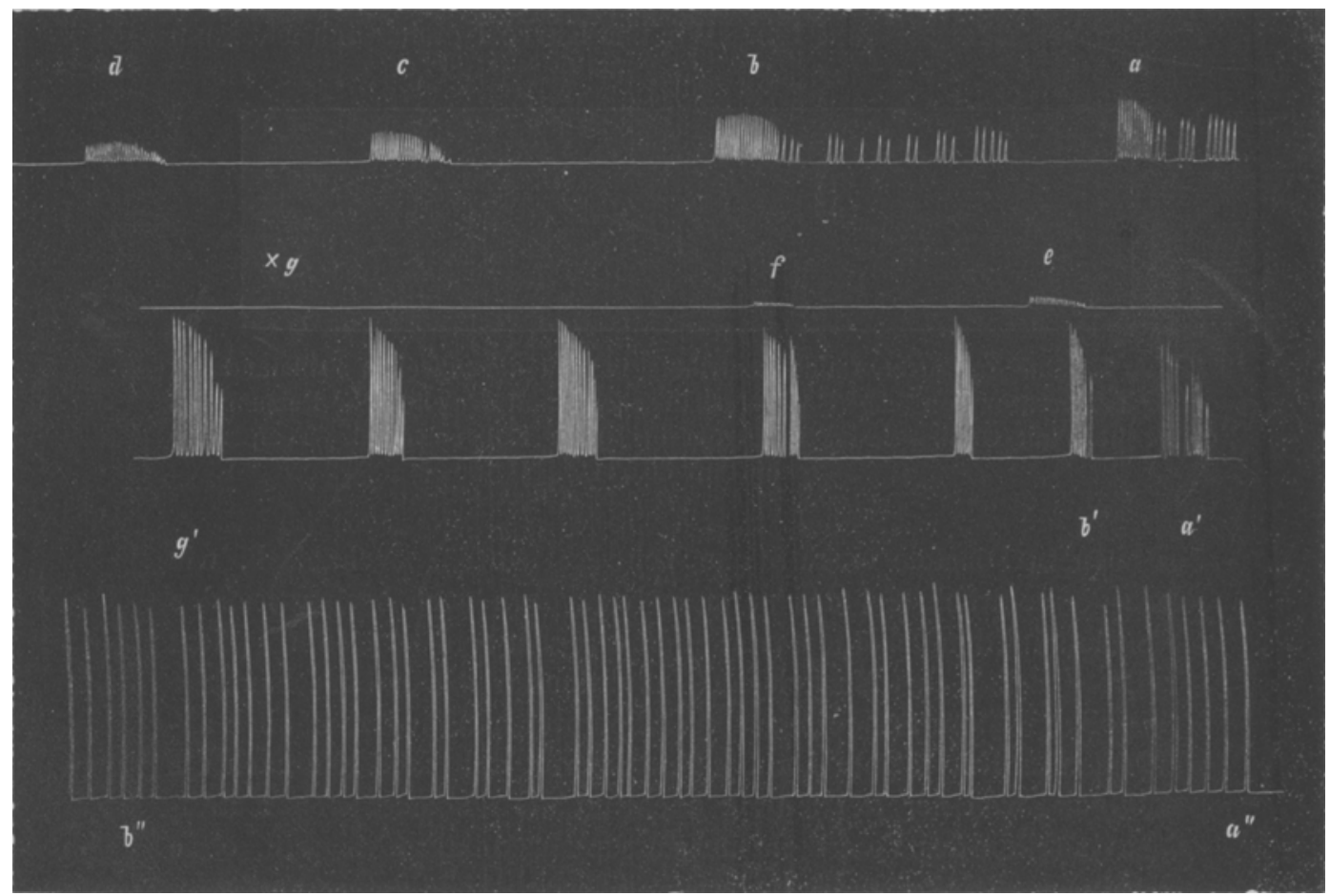

Knrve 2b. Kurz vor a Anlegen der Stanniusschen Ligatur zwischen Vorhöfen und Ventrikel. Bei $a$ und $b$ noch deutliche mechanische und elektrische Reizbarkeit bei $5 \mathrm{~cm}$ Rollenabstand. (Die mehr auseinanderstehenden Perioden bedeuten die mechanischen, die engen die elektrischen Reize.) Von $e$ bis f Abnehmen der Reizbarkeit. Beig spricht der Ventrikel weder auf mechanische, noch auf elektrische Reize mehr an. Temperatur konstant $38^{\circ} \mathrm{C}$. $a^{\prime}$ bis $g^{\prime}$ Wiederherstellung der mechanischen und elektrischen Reizbarkeit schon bei Temperaturen, die zum Wiedereinsetzen der regnlären Ventrikeltätigkeit noch nicht geniigen. $a^{\prime \prime} b^{\prime \prime}$. Fast völlig normale Ventrikelfunktion auf Zusatz von

2 Tropfen Adrenalin 1:1000 unmittelbar nach der Reizung bei $g^{\prime}$. 
gleichbleibender Temperatur, minutenlang danernde Perioden regelmäßiger Herzatätig:keit auszulösen vermag (s. Kurve 3 ).

2. Über den Würmestillstand des Stanniusherzens.

Lehren die angefübrten Befunde, nämlich die zumeist unveränderte Tätigkeit von Sinus und Vorhöfen bei stillstehendem Ventrikel und die meist unverminderte mechanische und elektrische Reizbarkeit der Kammer, daß die Reizerzeugung und die Ventrikelmuskulatur beim Einsetzen des Wärmeschocks noch so gut wie intakt geblieben sind, während die atrioventrikuläre Reizleitung durch Erwärmung unterbrochen wurde, so beweisen Versuche an Herzen, die nach Einsetzen des Ventrikelstillstandes durch Stanniussche Ligatur an der Atrioventrikulargrenze unterbunden worden waren, daß die autonomen motorischen Kammerzentren - im Augenblick der eintretenden Schädigung funktionsfäbig geblieben und durch Adrenalin, welches nach Gottlieb(13) hauptsächlich diese Apparate zu. erregen vermag, wieder zu nahezu regelmäßiger Tätigkeit za bringen sind ( $\mathrm{s}$. Kurve $2 \mathrm{~b}$ ).

3. Versuche, den Wärmestillstand durch Gifte zu beheben.

Bereits Schelske hatte beobachtet, daß die durch Wärme stillstehende Kammer des Froschherzens bei Vagusreizung wieder zum Schlagen gebracht werden kann, aber erst Stewart (14) hat in einwandfreier Weise gezeigt, daß bei sorgfältigst ausgeführter Vagusreizung der eben in den Wärmestillstand eingetretene Ventrikel für kürzere Zeit in mehr oder weniger regelmäßigen Gang zu setzen ist,

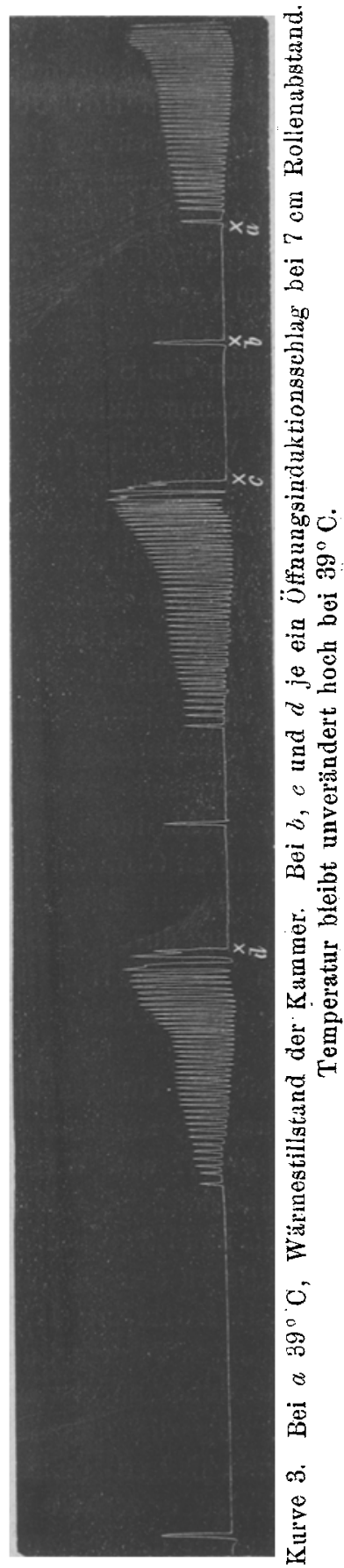


während Sinus und Vorhöfe infolge der Vagusreizung stillstehen, welches Spiel mehrfach wiederholt werden kann. Durch Untersuchungen von Rothberger und Winterberg (15) wissen wir, daß das Unterbleiben des vagalen Hemmungseffektes auf die Kammer bei faradischer Reizung des Vagus unter dem Einfluß der verschiedensten Gifte dadurch bedingt ist, daB bei eingetretener Reizleitungsunterbrechung durch diese Gifte die Kammerantomatie durch Reizung teilweise im Vagus verlaufender Acceleransfasern geweckt wird. Allerdings ist hier zu bemerken, daß Loewi (16) weder am Frosch, noch beim Kaninchen *im Stadium der Vaguslähmung " durch Maskarin und Pilokarpin Kammerautomatie hat hervorrufen können. Schon aus den Versuchen von Schelske und Stewart geht daher unseres Erachtens hervor, da $\boldsymbol{B}$ zur Zeit des begonnenen Wärmestillstandes der Kammer eine funktionelle Unterbrechung zwischen Vorhöfen and Ventrikel bei gleichzeitig erhaltener Reizbarkeit des automatischen motorischen Ventrikelapparates besteht. Es lag daher nahe, zu prüfen, inwieweit Gifte, welche vorwiegend auf die Kammerautomatie einwirken, das in Wärmenarkose diastolisch stillstehende Herz wieder zum Schlagen zu bringen vermögen. Naturgemäß ist - bei derartigen Versuchen darauf zu achten, daß einerseits die Muskulatur mechanisch und elektrisch erregbar; andererseits aber 'der Wärmestillstand doch so ausgeprägt ist, daß er nicht durch spontane Pulsationen, welche einen Effekt der angewandten Gifte vortäuschen könnten, gestört wird. Endlich mußte man sich am Ende jedes Versuches davon îberzengen, ob die Schädigung durch Abkühlung tatsäehlich auch reversibel war. 'Wir priften Adrenalin, Physostigmin, Atropin, Strophanthin, Spartein und Baryt:

a) Adrenalin.

Seit den Untersuchungen Gottliebs ist bekannt, daß das Adrenalin auf die automatischen motorischen Ventrikelzentren wirkt, und Rothberger und Winterberg (15) zeigten am Elektrokardiogramm des Hundeherzens, daß Adrenalin ähnlich wie Muskarin, Physostigmin, Digitalis und Strychnin in mehrminder ausgeprägter Weise die Kammerautomatie zu erregen vermag. Hierher gehört auch die Beobachtung von F. B. Hofmann (17), daB Adrenalin häufig den von den Vorhöfen ahgetrennten Ventrikel des Froschherzens wieder zum Schlagen bringt. Es war daher zu erwarten, daß auch bei einsetzendem Wärmestillstand, fälls die automatischen Ventrikelzentren dabei intakt sind, Adrenalin das stillstehende Herz zu einer mehrminder regelmäßigen Schlagfolge erwecken kann. Da dies jedoch von der jeweiligen Erregbarkeit der in Betracht kommenden nervösen Apparate abhängig 
und eine scharfe Abgrenzung der Wärmeschädigung sebr schwer ist, so ist rom Adrenalin nicht in jedem Falle ein sicherer. Effekt zu erwarten. Kurve $2 b\left(a^{\prime \prime}-b^{\prime \prime}\right)$ zeigt, wie der elektrisch gut erregbare Ventrikel auf zwei Tropfen Adrenalin $1: 1000$ in nahezu regelmäBige automatische Schlagfolge zu versetzen ist. Einen ähnlichen Effekt weist Kurve 4 auf.

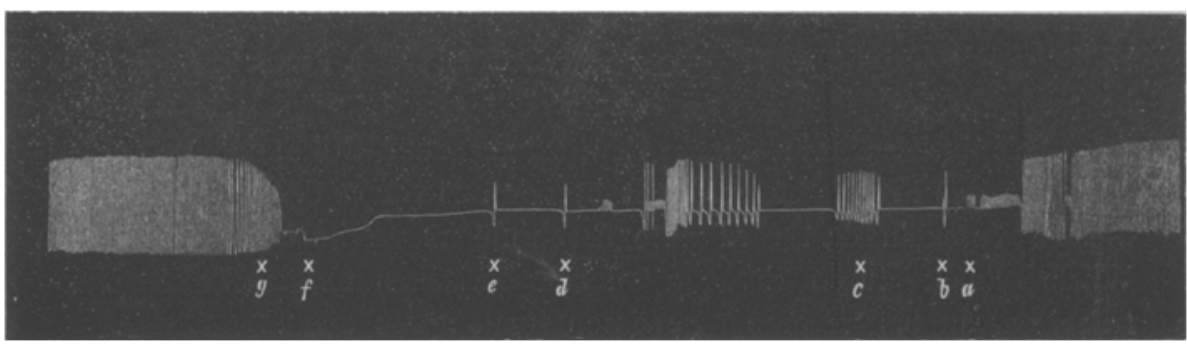

Kurve 4. Bei $a 39^{\circ} \mathrm{C}$, Wärmestillstand. $b$ Mechanische, $c$ elektrische Reizung (Induktionsöffnungsschläge bei $6 \mathrm{~cm}$ Rollenabstand). Uṇmittelbar danach 2 Tropfen Adrenalin 1:1000. $d$ und $e$ Mechanische Reize. Temperatur bisher $39^{\circ} \mathrm{C}$. $f$ Abkïhlung. $g 32^{\circ} \mathrm{C}$.

b) Physostigmin.

Die Wirkung des Physostigmins anf den durch Wärme diastolisch stillgestellten Ventrikel ist der des Adrenalins ganz ähnlich (s. Kurve 5).

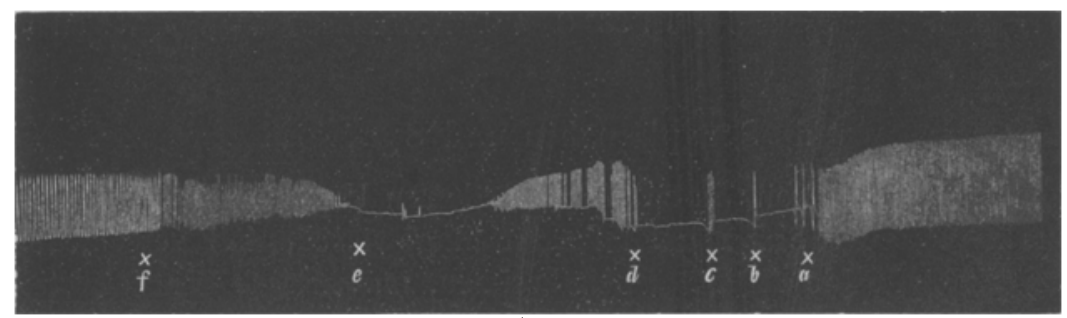

Kurve 5. $a \cdot 38^{\circ} \mathrm{C}$. Einsetzen des Ventrikelstillstandes. $b$ Méchanische Reizung $c$ Mehrere Induktionsöffnungsschlige bei $5 \mathrm{~cm}$ Rollenabstand. $d$ 0,3 Physostigmin 1\%0. Temperatur konstant $38^{\circ} \mathrm{C}$. Bei e Abkiihlung. $f 30^{\circ} \mathrm{C}$.

Der Einfuß des Physostigmins auf das Herz wurde von Harnack und Witkowsky (18) durch Erregung der Muskelsubstanz erklärt. Es ist indessen wahrscheinlicher, daß die von uns am durch Wärme stillgestellten Ventrikel beobachtete teilweise Wiederherstellung seiner Funktion durch Physostigmin nicht sowohl durch Steigerung der Erregbarkeit der Muskulatur, als vielmehr durch Einwirkung anf die automatischen Kammerapparate erzielt wurde (vgl. Luchsinger 21). 


\section{c) Atropin.}

Bekanntlich besteht neben der iberwiegenden, den Herzhemmungsapparat lähmenden Atropinwirkung noch eine die motorischen antomatischen Apparate des Ventrikels erregende, wie schon Untersuchungen von Boehm(19), Roßbach (20), Luchsinger (21), Langendorf (22) u. a. dargetan haben. So ließen sich z. B. Herzen, die durch Akonitin, Blausäure, Chinin usw., oder durch die Stanniussche Ligatur zum Stillstand gebracht worden waren, wieder, wenn auch nur für eine beschränkte Zeit, durch Atropin zum Schlagen bringen. Die Wirkung des Atropins auf die durch Erwärmung zum Stillstand gebrachte Kammer dẹs Froschberzens geht hervor aus Kurve 6.' Man sieht aus diesem Versuch, daB verdünnte Atropinlösungen imstande sind, den Wärmeschock durch Erregung der motorischen automatischen Ventrikelzentren bis zu einem gewissen Grad aufzuheben.

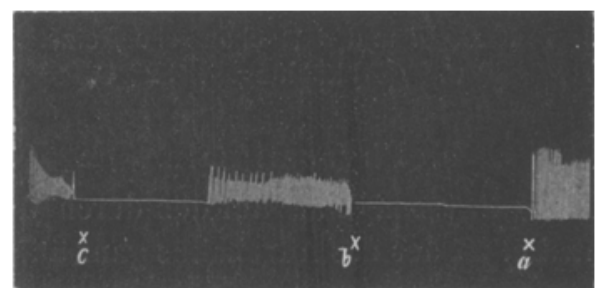

Kurve 6. $a$ Würmestillstand bei $38^{\circ}$ C. $\quad b .0,2$ Atropin. sult. 5\% $\%$. c. Abkühlung.

\section{d) Strophanthin und Spartein.}

Es wurde bereits in zahlreichen älteren Untersuchungen (Knoll, Cushny; Brandenburg 23) darauf hingewiesen, daß verschiedene Digitalisglykoside die Fähigkeit besitzen, im Verlaufe der Vergiftung die. Herzkammer unabhängig von den Vorhöfen zum Schlagen zu bringen. Rothberger and Winterberg(24), haben durch ansgedehnte Versuche an Hundeherzen mittels des Elektrokardiogramms gezeigt, daB das Strophanthin die Reizbildung in den automatischen Ventrikelzentren gtinstig beeinflussen kann, wenn auch schwächer, als die den Digitalisglykosiden in vielfacher Beziehung so analogen Bariumsalze. Es war daher anzunehmen, daß auch der in Wärmestillstand geratene Ventrikel des Froschherzens durch Strophanthin zu automatischer Tätigkeit angeregt wïrde. Die von uns mit Strophanthin ausgeführten Versuche lehren, daß in der Tat dieses Glykosid eine, wenn auch geringfügige Wirkung in diesem Sinne austubt 
(Kurve 7). Ganz ähnlich verhält es sich mit Spartein, von welchem allerdings viel größere Dosen nötig sind (vgl. Kurve 8).

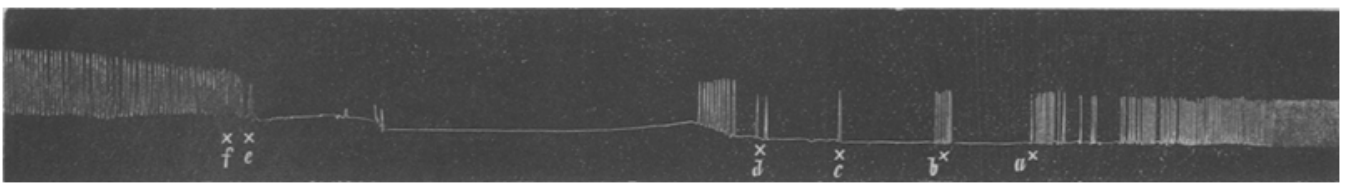

Kurve 7. $a$ Einsetzen des Wärmestillstandes bei $38^{\circ} \mathrm{C} . \quad b$ Öffnungsinduktionsschläge bei $5 \mathrm{~cm}$ Rollenabstand. $c$ Mechanischer Reiz. Bei $d$ 0,2 Strophanthin $0,1 \%$ \%. e Abkühlung. Bei $f 34^{\circ} \mathrm{C}$.
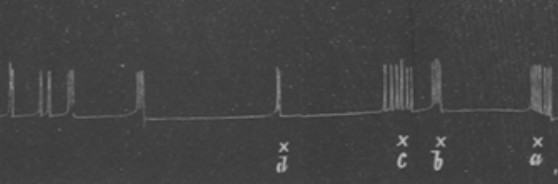

Knrve 8. $a 38,5^{\circ} \mathrm{C} . \quad b$ Gruppe von Öffnungsinduktionsschlägen bei $7 \mathrm{~cm}$ Rollenabstand. $c$ Mechanische Reize. Bei $d 1 \mathrm{ccm} 1 \%$ iges Spartein. e Abkühlung. $732^{\circ} \mathrm{C}$.

e) Baryt.

Im Anschluß an anderweitige Untersuchungen über die Einwirkung von Barium auf das stillstehende Herz, welche A. Fröhlich gemeinsam mit dem einen von uns (P.) ausgefubrt hat 1), und die nach Analogie der Experimente vón Rothberger und Winterberg(25) die ganz auffallende Empfindlichkeit der automatischen motorischen Kammerzentren fur Bariumsalze aufs neue zeigen konnten, haben anch wir an den in Wärmenarkose stillstehenden Herzen untersucht, inwieweit Chlorbarium den Wärmeschock beheben kann. Rotbberger und Winterberg fanden bereits nach intravenöser Injektion von 0,5 bis 1. ecm einer $10 \%$ igen Chlorbariumlösung an Hunden die Entwicklung einer spontanen Kammerautomatie. In unseren Versuchen zeigte es sich, daß bereits Mengen von $\frac{1}{100000000}$ g Chlorbarium eine mehrminder regelmäßige automatische Ventrikeltätigkeit auslösen können (s. Kurve 9), ein Beweis, wie uns scheint, dafür, daß der Wärmestillstand zunächst die automatischen Kammerapparate und die Muskulatur intakt läßt und nur die Reizleitung schädigt. Daraus war zu folgern, daß die Darreichung von Baryt vor dem Wärmestillstand einen gewissen Schutz gegen diesen bilden würde, indem nach Versagen der Reizleitung die Kammerautomatie zur Entfaltung käme und sich

1) Erscheint in diesem Archiv. 


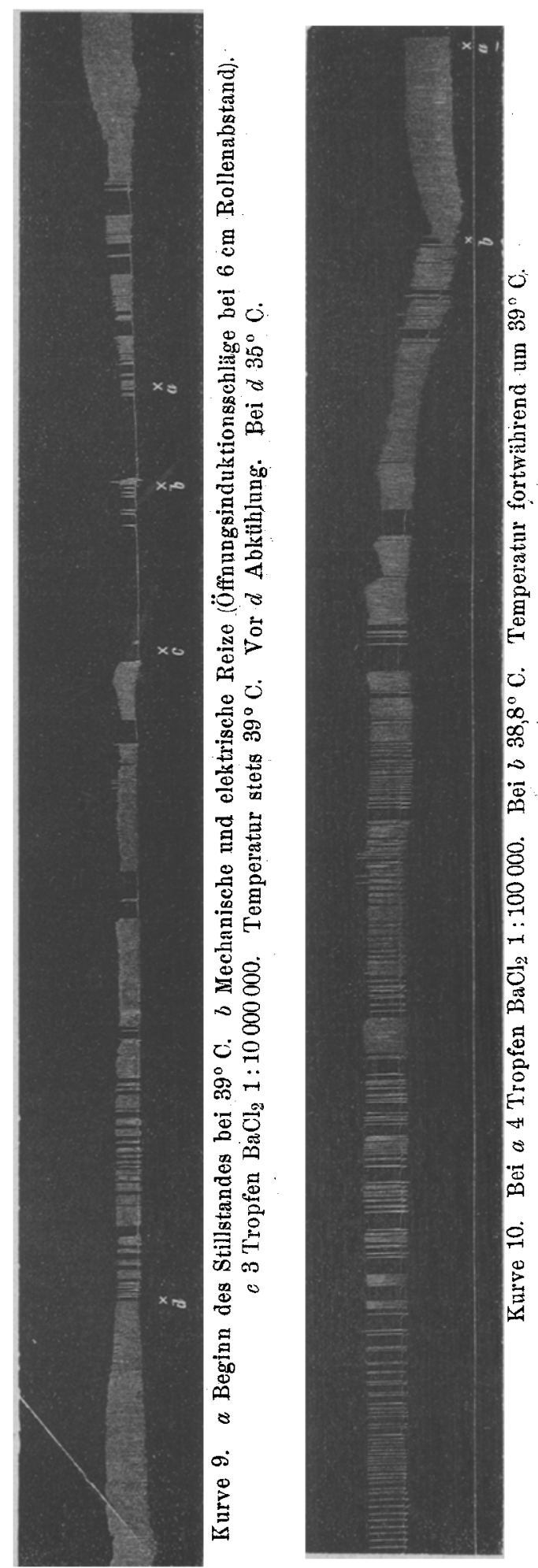

trotz weiter bestehender Erwärmung eine mehrminder regelmäßige Herzfunktion einstellen würde. In den darauf gerichteten Versuchen (Kurve 10) ließ sich nachweisen, daB Temperaturen, die sonst den Wärmeschock auslösen, insofern ohne EinfluB sind, als nach Versagen der Reizleitung unmittelbar die Ventrikelantomatie einsetzt. In der angeführten Kurve ist ersichtlich, daß die auf den Ventrikel fortgeleitete Vorhofstätigkeit aussetzt, und an deren Stelle das unter Barytwirkung stehende Herz automatisch zu schlagen beginnt. Gleichzeitig sieht $\operatorname{man}^{\prime}$ eine von dieser, die atomatischen Zentren anregenden Barytwirkung scheinbar unabhängige zweite auf die Muskulatur des Ventrikels, indem sich diese der Systole nähert. Hier sei noch daran erinnert, daß bereits Boehm (26) ein Versagen der Vagushemmung nach Einwirkung von Bariumsalzen beobachtet hat und auch sah, daß der Muskarinstillstand durch Baryt definitiv aufgehoben werden kann. 
Die vorstehenden, mit Adrenalin Ptyssostigmin, Atropin, Strophanthin und Spartein, insbesondere wher nait Baryt durchgeführten Versuche lehren, daß der diastolische Wärmestillstand des Herzens den gesamten nervösen und muskulären Ventrikelapparat wenigstens in den ersten Stadien mehrweniger intakt läßt; so daß als Ursache des durch Wärmenarkose bedingten Herzstillstandes in Diastole in erster Linie eine Störung des atrioventrikulären Übergangssystems angenommen werden muß. Es liegt in der Natur der Wärmeschädigung, daß sie sich bei längerer Einwirkung (individuelle Versehiedenheiten der Herzen) nicht scharf auf die Reizleitung allein begrenzen läßt, sondern daß allmählich auch die automatischen motorischen Zentren und die Erregbarkeit der Ventrikelmuskulatur in Mitleidenschaft gezogen werden. Hier und da sieht man endlich, besonders bei'Temporarien, daß auch die Reizerzeugung durch die Wärme geschädigt wird, so daß dann der ganze Herzmechanismus außer Funktion gesetzt ist. Hält sich jedoch die Schädigung innerhalb der für die Wärmenarkose gesetzten Grenzen, d. h. also zwischen 38 und $39,5^{\circ} \mathrm{C}$, so gelingt es immer, sämtliche Herzfunktionen durch Abkühlen wieder herzustellen. Erster Angriffspunkt der Schädigung und Ursache des diastolischen Ventrikelstillstandes bleibt aber immer das Reizleitungssystem, welches den fiir die Erwärmung empfindlichsten Teil des Herzapparates darstellt. Die Analyse der Wärmenarkose zeigt zahlreiche Analogien zur Lichtschädigung des isolierten Froschherzens. Auch dort sehen wir den Lichtsehock bedingt vor allem durch das Versagen des Überleitungsapparates. Der Hauptuntersehied zwischen beiden Schädignngen ist der, daß es sich hier, dem Wesen der Narkose entsprechend, um eine völlig reversible Erscheinung handelt, während die Lichtschädigung so gut wie immer irreversibel bleibt.

\section{Schlußsätze.}

1. Der Wärmestillstand des Froschherzens charakterisiert sich durch das diastolische Aussetzen der Kammer, während Sinus und Vorhöfe noch weiterschlagen.

2.. Die durch Wärme diastolisch stillgestellte Kammer des intakten sowohl, wie des nach Stannius an der Vorhofkammergrenze eingeschnürten Herzens, bleibt mechanisch und elektrisch erregbar.

3. Der ron Schelske und Stewart beobachtete Reizerfolg an der dureh Wärme stillgestellten Kammer bei faradischer Reizung des Vagus und Sympathikus beruht auf 
Erregung der Kammerautomatie bei aufgehobener Reizleitung.

4. Die Kammerautomatie des durch Wärme stillgestellten Herzens kann in mäBigem G'rade angeregt werden durch Adrenalin, Physostigmin, Atropin,Strophanthin und Spartein. In mächtiger Weise dagegen wirkt Baryt. Bereits Dosen von 1 $1 \overline{00000000} \mathrm{~g}$ versetzen den Ventrikel in automatische, mehrminder regelmäßige Tätigkeit. Schon bei nurwenig größ̣eren Dosen zeigt sich neben der Wirkung auf die automatiséhen Zentren die zur systolischen Kammerstellung führende tonische Wirkung auf die Muskulatur.

5. Es gelingt mitunter, durch vorherige Darreichung: geringer Barytdosen den Wärmestillstand hintanzubalten.

6. Man kann demnach den durch Temperaturerhöhung erzeugten diastolischen Stillstand des isolierten Froschherzens im wesentlichen als Wärmenarkose des atrioventrikulären Reizleitungssystems auffassen.

\section{Literatur.}

1. Cl. Bernard, zitiert nach Richet, Dict. de physiologie, III., S. 237 und 253. - 2. H. H. Meyer, Über die Beziehung zwischen den Lipoiden und pharmakologischer Wirkung. Münch. medizin. Wochensehrift Nr. 31, S. 1577, 1909. - 3. Schelske, Über die Veränderungen der Erregbarkeit durch die Wärme. Heidelberg 1860. - 4. Cyon, Über den Einfluß der Temperaturveränderungen anf Zahl, Dauer und Stärke der Herzschlïge. Lndwigs Arbeiten S. $256 \mathrm{ff}, 1886$. - 5. Aristow, Einfluß plötzlichen Temperaturwechsels auf das Herz usw. Du Bois-Reymonds Arch. f. Phys. S. 198, 1879. - 6. Ludwig und Luchsinger, Die Wirksamkeit des Nervus vagus als Funktion der Temperatur. PAlügers Arch. Bd. 25, S. 211. - 7. S tewart, The influence of temperature on the heart etc. Journ. of physiology, X̌III., S. 59. - 8. Ide, Wie erklïrt sich der Stillstand des ïbererwärmten Herzens? Du Bois-Reymonds Arch. f. Phys. S. 243, 1892. - 9. Unger, Über den Würmestillstand des Froschherzens. Pflügers Arch. Bd. 149, S. 364. - 10. A msler und́ Pick, Pharmakologisebe Untersuchungen über die biologische Wirkang des Flnoreszenzlichtes am isolierten Froschberzen. Areh. f. exper. Path. 1. Pharm. Bd. 82, S. 85. - 11. Fla tow, Über den Einfluß der Temperatur auf die Tätigkeit des Froschherzens. Ebenda Bd. 30, S. 363. - 12. Straub, Zur Physiologie des Aplysienherzens. Pftügers Arch. Bd. 86, S. 524. - 13. Gottlieb, Über die Wirkung der Nebennierenextrakte auf Herz und Blutdruck. Arch. f. exper. Path. u. Pharm. Bd. 38, S. 99. - 14. Stew art, Einfluß der Herztemperatur auf die 'Tätigkeit der Hemmungsnerven des Herzens. Zeitschr. f. Biol. Bd. 59. S. 531. - 15. Rothberger und Winterberg, Über seheinbare Vagnslïhmung bei Muskarin, Physostigmin und anderen Giften, sowie bei intrakardialer Drucksteigerang. Pflïgers Arch. Bd. 132, S. 233. Ferner: Dieselben, Ebenda Bd. 135, S. 559. - 16. Loewi, 0., Unter- 
suchungen zur Physiologie und Pharmakologie des Herzvagns. III. Mitteilung: Vaguserregbarkeit und Vagusgifte. Arch. f. exper. Path. u. Pharm. Bd. 70, S. 351. - 17. Hofmann und Holzinger, Über den Einfluß von Extrasystolen auf die Rhythmik spontan schlagender Herzteile. Zeitschr. f. Biol. Bd. 57, S. 313. - 18. Harnack und Witkowski, Pharmakologische Untersuchungen iiber Physostigmin und Calabarin. Arch. f. exper. Path. u. Pharm. Bd. 5, S. 401. 19. B oe hm, Studien über Herzgifte S. 31, 1871. - 20. Roßbach und Papilsky, Pharmakologische Untersuchungen Bd. 2, S. 129. - 21. Luchsinger, Eine toxikologische Versuchsreihe. Arch. f. exper. Path. п. Pharm. Bd. 14, S. 370. 22. Langendorff, O., Stndien über Rhythmik und Antomatie des Froschherzens. Du Bois-Reymonds Arch. f. Phys. 1884, Supplementband. Siehe auch Harnack und Hafemann, Pharmakologisehe Studien am isolierten Froschherzen mit besonderer Beriicksichtigung des Atropins und des Kupfers. Daselbst auch die ältere Literatur. Arch. f. exper. Path. u. Pharm. Bd. 17, S. 145. - 23. Knoll, Cushny, Brandenburg, zitiert nach Rothberger und Winterberg, Pfligers Arch. Bd. 150, S. 218. - 23. Rothberger und Winterberg, Über den Einfuß von Strophanthin auf die Reizbildungstätigkeit der automatischen Zentren des Herzens. Ebenda Bd.150, S. 217. - 25. Dieselben, Über die experimentelle Erzengung extrasystolischer ventrikulärer Tachykardie durch Acceleransreizung (ein Beitrag zur Herzwirkung von Barium and Kalzium). Ebenda Bd. 142, S. 461. - 26. B o ehm, Über die Wirkung von Barytsalzen auf den Tierkörper usw. Arch. f. exper. Path. u. Pharm. Bd. 3 , S. 216. 